\title{
Drones in California research and extension
}

Maggi Kelly, Professor and UC Cooperative Extension Specialist, UC Berkeley, and Director, Statewide IGIS Program

$\mathrm{F}$ or more than 150 years, people have been taking aerial photos of the Earth. In the early days, cameras loaded with panchromatic film were mounted on kites, balloons and the occasional unfortunate pigeon. Today we have over 100 operational earth observation satellites in orbit owned by individual countries and private companies. These satellites collect digital imagery from a broad range of the electromagnetic spectrum beyond visible light, and at spatial resolutions from 1

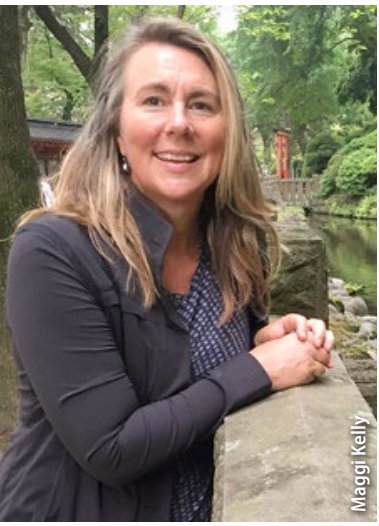

Maggi Kelly kilometer down to 1 meter. This global image archive has provided a 40-year record of how our crops, cities and natural landscapes change and function in response to seasons, management and disturbances.

While this source of digital earth observation has been incredibly useful, satellite imagery presents certain challenges. The pixel size of typical satellite imagery can be too large for finescale ecological or agricultural research. The timing of satellite overflights does not always synchronize with research or management needs, and the cost of proprietary imagery can be prohibitive.

UAS, or unmanned aerial systems, commonly referred to as drones, can address these spatial, temporal and cost-related challenges.

We have heard about drones as nuisances: the 2015 "drunk droning" case at the White House, or hobbyists interfering with firefighting aircraft, etc. However, drones have also been labeled as democratizers of scientific data collection because they allow for timely, responsive, highly detailed and relatively cheap imagery collection.

Both of these views of drones are true.

First, the good news: drones are revolutionizing the way we collect data about agricultural and natural resources, and the University of California is poised to be a leader in this emerging field.

For scientific research, we typically use small rotorcraft that can cover 50 to 200 acres per 15- to 25-minute flight. Cameras mounted on these drones range from true-color digital cameras to multispectral cameras that are ideal for mapping vegetation. Drones can also carry cameras sensitive to thermal radiation, and LIDAR sensors. From sets of overlapping drone images, we can create high-resolution digital elevation models (DEMs), 3-D models of structures or vegetation, and high-resolution digital image mosaics.

The applications for drones in California are broad and farreaching. Drone imagery has been used to map invasive weeds in agricultural fields, measure critical aspects of ecosystem function such as vegetation dynamics, water status and plant productivity, and to monitor animal population ecology and biodiversity inventories. Meanwhile, DEMs have been used to evaluate canopy structure and micro-topography important to soil moisture, and drone-created 3-D models are being used to map campus infrastructure and individual trees with detail not seen before.

While revolutionary, drones also come with challenges.

Drone flight in commercial airspace is highly regulated for researchers, while hobbyists fly with abandon to collect personal imagery (witness the profusion of "drone selfies" on YouTube). Flying drones for work, business purposes, or for compensation or hire means that certain steps must be taken before flying. These are detailed in the accompanying article by Sean Hogan et al. (page 6) that addresses drone registration and a remote pilot certification.

\section{A California framework for drone science and outreach}

There are numerous examples of drone data supporting science and outreach across UC. These are largely individual projects that are not connected. Now more than ever, we need a collective effort to support drones at scale throughout UC and California. This vision builds on the broad base of applications already evident in the UC system, and creates a framework for science, education and entrepreneurship around practical large-scale drone implementations. To build this, we need to (1) utilize UC's broad technical expertise in open data repositories, big data workflows and data synthesis, (2) expand on the ability of UC to be a partner and incubator to local businesses, (3) develop more application-specific use cases from our network of living laboratories - the UC Natural Reserve System and the UC ANR Research and Extension Center System - and (4) leverage UC ANR's long experience in community-engaged science and outreach.

Drones represent an important strategic opportunity for UC and for California. By supporting this technology, the sky is the limit! CA

\section{References}

Anderson K, Gaston KJ. 2013. Lightweight unmanned aerial vehicles will revolutionize spatial ecology. Front Ecol Environ 11:138-46.

Baldocchi D, Falge E, Gu L, et al. 2001. FLUXNET: A new tool to study the temporal and spatial variability of ecosystem-scale carbon dioxide, water vapor, and energy flux densities. B Am Meteorol Soc 82:2415-34.

Wulder MA, Masek JG, Cohen WB, et al. 2012. Opening the archive: How free data has enabled the science and monitoring promise of Landsat. Remote Sens Environ 122:2-10. 